CERN-PPE/95-184

$13^{\text {th }}$ December 1995

\title{
Quark and Gluon Jet Properties in Symmetric Three-Jet Events
}

\author{
The ALEPH Collaboration
}

\begin{abstract}
Quark and gluon jets with the same energy, $24 \mathrm{GeV}$, are compared in symmetric three-jet configurations from hadronic $Z$ decays observed by the ALEPH detector. Jets are defined using the Durham algorithm. Gluon jets are identified using an anti-tag on $b$ jets, based on either a track impact parameter method or a high transverse momentum lepton tag. The comparison of gluon and mixed flavour quark jets shows that gluon jets have a softer fragmentation function, a larger angular width and a higher particle multiplicity. Evidence is also presented which shows that the corresponding differences between gluon and heavy flavour jets are significantly smaller.
\end{abstract}

Submitted to Phys.Lett.B 


\section{The ALEPH Collaboration}

D. Buskulic, D. Casper, I. De Bonis, D. Decamp, P. Ghez, C. Goy, J.-P. Lees, A. Lucotte, M.-N. Minard, P. Odier, B. Pietrzyk

Laboratoire de Physique des Particules (LAPP), IN P $^{3}$-CNRS, 74019 Annecy-le-Vieux Cedex, France

M. Chmeissani, J.M. Crespo, I. Efthymiopoulos, E. Fernandez, M. Fernandez-Bosman, Ll. Garrido, ${ }^{15}$ A. Juste, M. Martinez, S. Orteu, A. Pacheco, C. Padilla, F. Palla, A. Pascual, J.A. Perlas, I. Riu, F. Sanchez, F. Teubert

Institut de Fisica d'Altes Energies, Universitat Autonoma de Barcelona, 08193 Bellaterra (Barcelona), Spain $^{7}$

A. Colaleo, D. Creanza, M. de Palma, A. Farilla, G. Gelao, M. Girone, G. Iaselli, G. Maggi, ${ }^{3}$ M. Maggi, N. Marinelli, S. Natali, S. Nuzzo, A. Ranieri, G. Raso, F. Romano, F. Ruggieri, G. Selvaggi, L. Silvestris, P. Tempesta, G. Zito

Dipartimento di Fisica, INFN Sezione di Bari, 70126 Bari, Italy

X. Huang, J. Lin, Q. Ouyang, T. Wang, Y. Xie, R. Xu, S. Xue, J. Zhang, L. Zhang, W. Zhao

Institute of High-Energy Physics, Academia Sinica, Beijing, The People's Republic of China ${ }^{8}$

R. Alemany, A.O. Bazarko, G. Bonvicini, ${ }^{23}$ M. Cattaneo, P. Comas, P. Coyle, H. Drevermann, R.W. Forty, M. Frank, R. Hagelberg, J. Harvey, R. Jacobsen, ${ }^{24}$ P. Janot, B. Jost, E. Kneringer, J. Knobloch, I. Lehraus, E.B. Martin, P. Mato, A. Minten, R. Miquel, Ll.M. Mir, ${ }^{2}$ L. Moneta, T. Oest, P. Palazzi, J.R. Pater, ${ }^{27}$ J.-F. Pusztaszeri, F. Ranjard, P. Rensing, L. Rolandi, D. Schlatter, M. Schmelling, O. Schneider, W. Tejessy, I.R. Tomalin, A. Venturi, H. Wachsmuth, T. Wildish, W. Witzeling, J. Wotschack

European Laboratory for Particle Physics (CERN), 1211 Geneva 23, Switzerland

Z. Ajaltouni, A. Barrès, C. Boyer, A. Falvard, P. Gay, C . Guicheney, P. Henrard, J. Jousset, B. Michel, S. Monteil, J-C. Montret, D. Pallin, P. Perret, F. Podlyski, J. Proriol, J.-M. Rossignol

Laboratoire de Physique Corpusculaire, Université Blaise Pascal, IN ${ }^{2} P^{3}-C N R S$, Clermont-Ferrand, 63177 Aubière, France

T. Fearnley, J.B. Hansen, J.D. Hansen, J.R. Hansen, P.H. Hansen, B.S. Nilsson, A. Wäänänen

Niels Bohr Institute, 2100 Copenhagen, Denmark ${ }^{9}$

A. Kyriakis, C. Markou, E. Simopoulou, I. Siotis, A. Vayaki, K. Zachariadou

Nuclear Research Center Demokritos (NRCD), Athens, Greece

A. Blondel, ${ }^{21}$ G. Bonneaud, J.C. Brient, P. Bourdon, A. Rougé, M. Rumpf, R. Tanaka, A. Valassi, ${ }^{6}$ M. Verderi, H. Videau ${ }^{21}$

Laboratoire de Physique Nucléaire et des Hautes Energies, Ecole Polytechnique, IN ${ }^{2} P^{3}-C N R S, 91128$ Palaiseau Cedex, France

D.J. Candlin, M.I. Parsons

Department of Physics, University of Edinburgh, Edinburgh EH9 3JZ, United Kingdom ${ }^{10}$

E. Focardi, G. Parrini

Dipartimento di Fisica, Università di Firenze, INFN Sezione di Firenze, 50125 Firenze, Italy

M. Corden, M. Delfino, ${ }^{12}$ C. Georgiopoulos, D.E. Jaffe

Supercomputer Computations Research Institute, Florida State University, Tallahassee, FL 323064052, USA $^{13,14}$

A. Antonelli, G. Bencivenni, G. Bologna, ${ }^{4}$ F. Bossi, P. Campana, G. Capon, V. Chiarella, G. Felici, P. Laurelli, G. Mannocchi, ${ }^{5}$ F. Murtas, G.P. Murtas, L. Passalacqua, M. Pepe-Altarelli

Laboratori Nazionali dell'INFN (LNF-INFN), 00044 Frascati, Italy 
L. Curtis, S.J. Dorris, A.W. Halley, I.G. Knowles, J.G. Lynch, V. O’Shea, C. Raine, P. Reeves, J.M. Scarr, K. Smith, A.S. Thompson, F. Thomson, S. Thorn, R.M. Turnbull

Department of Physics and Astronomy, University of Glasgow, Glasgow G12 8QQ,United Kingdom ${ }^{10}$

U. Becker, O. Braun, C. Geweniger, G. Graefe, P. Hanke, V. Hepp, E.E. Kluge, A. Putzer, B. Rensch, M. Schmidt, J. Sommer, H. Stenzel, K. Tittel, S. Werner, M. Wunsch

Institut für Hochenergiephysik, Universität Heidelberg, 69120 Heidelberg, Fed. Rep. of Germany ${ }^{16}$

D. Abbaneo, R. Beuselinck, D.M. Binnie, W. Cameron, D.J. Colling, P.J. Dornan, A. Moutoussi, J. Nash, G. San Martin, J.K. Sedgbeer, A.M. Stacey

Department of Physics, Imperial College, London SW7 2BZ, United Kingdom ${ }^{10}$

G. Dissertori, P. Girtler, D. Kuhn, G. Rudolph

Institut für Experimentalphysik, Universität Innsbruck, 6020 Innsbruck, Austria $^{18}$

C.K. Bowdery, T.J. Brodbeck, P. Colrain, G. Crawford, A.J. Finch, F. Foster, G. Hughes, T. Sloan, E.P. Whelan, M.I. Williams

Department of Physics, University of Lancaster, Lancaster LA1 4YB, United Kingdom ${ }^{10}$

A. Galla, A.M. Greene, K. Kleinknecht, G. Quast, B. Renk, E. Rohne, H.-G. Sander, P. van Gemmeren C. Zeitnitz

Institut für Physik, Universität Mainz, 55099 Mainz, Fed. Rep. of Germany ${ }^{16}$

J.J. Aubert, ${ }^{21}$ A.M. Bencheikh, C. Benchouk, A. Bonissent, ${ }^{21}$ G. Bujosa, D. Calvet, J. Carr, C. Diaconu, F. Etienne, N. Konstantinidis, D. Nicod, P. Payre, D. Rousseau, M. Talby, A. Sadouki, M. Thulasidas, K. Trabelsi

Centre de Physique des Particules, Faculté des Sciences de Luminy, IN ${ }^{2} P^{3}$-CNRS, 13288 Marseille, France

I. Abt, R. Assmann, C. Bauer, W. Blum, D. Brown, ${ }^{24}$ H. Dietl, F. Dydak, ${ }^{21}$ G. Ganis, C. Gotzhein, K. Jakobs, H. Kroha, G. Lütjens, G. Lutz, W. Männer, H.-G. Moser, R. Richter, A. Rosado-Schlosser, S. Schael, R. Settles, H. Seywerd, R. St. Denis, W. Wiedenmann, G. Wolf

Max-Planck-Institut für Physik, Werner-Heisenberg-Institut, 80805 München, Fed. Rep. of Germany ${ }^{16}$

J. Boucrot, O. Callot, A. Cordier, M. Davier, L. Duflot, J.-F. Grivaz, Ph. Heusse, M. Jacquet, D.W. Kim, ${ }^{19}$ F. Le Diberder, J. Lefrançois, A.-M. Lutz, I. Nikolic, H.J. Park, ${ }^{19}$ I.C. Park, ${ }^{19}$ M.-H. Schune, S. Simion, J.-J. Veillet, I. Videau

Laboratoire de l'Accélérateur Linéaire, Université de Paris-Sud, IN² $\mathrm{P}^{3}$-CNRS, 91405 Orsay Cedex, France

P. Azzurri, G. Bagliesi, G. Batignani, S. Bettarini, C. Bozzi, G. Calderini, M. Carpinelli, M.A. Ciocci, V. Ciulli, R. Dell'Orso, R. Fantechi, I. Ferrante, L. Foà, ${ }^{1}$ F. Forti, A. Giassi, M.A. Giorgi, A. Gregorio, F. Ligabue, A. Lusiani, P.S. Marrocchesi, A. Messineo, G. Rizzo, G. Sanguinetti, A. Sciabà, P. Spagnolo, J. Steinberger, R. Tenchini, G. Tonelli, ${ }^{26}$ C. Vannini, P.G. Verdini, J. Walsh

Dipartimento di Fisica dell'Università, INFN Sezione di Pisa, e Scuola Normale Superiore, 56010 Pisa, Italy

A.P. Betteridge, G.A. Blair, L.M. Bryant, F. Cerutti, J.T. Chambers, Y. Gao, M.G. Green, D.L. Johnson, T. Medcalf, P. Perrodo, J.A. Strong, J.H. von Wimmersperg-Toeller

Department of Physics, Royal Holloway \& Bedford New College, University of London, Surrey TW20 OEX, United Kingdom ${ }^{10}$

D.R. Botterill, R.W. Clifft, T.R. Edgecock, S. Haywood, M. Edwards, P. Maley, P.R. Norton, J.C. Thompson

Particle Physics Dept., Rutherford Appleton Laboratory, Chilton, Didcot, Oxon OX11 OQX, United Kingdom ${ }^{10}$ 
B. Bloch-Devaux, P. Colas, S. Emery, W. Kozanecki, E. Lançon, M.C. Lemaire, E. Locci, B. Marx, P. Perez, J. Rander, J.-F. Renardy, A. Roussarie, J.-P. Schuller, J. Schwindling, A. Trabelsi, B. Vallage

CEA, DAPNIA/Service de Physique des Particules, CE-Saclay, 91191 Gif-sur-Yvette Cedex, France ${ }^{17}$

R.P. Johnson, H.Y. Kim, A.M. Litke, M.A. McNeil, G. Taylor

Institute for Particle Physics, University of California at Santa Cruz, Santa Cruz, CA 95064, USA ${ }^{22}$

A. Beddall, C.N. Booth, R. Boswell, C.A.J. Brew, S. Cartwright, F. Combley, A. Koksal, M. Letho, W.M. Newton, C. Rankin, J. Reeve, L.F. Thompson

Department of Physics, University of Sheffield, Sheffield S3 7RH, United Kingdom ${ }^{10}$

A. Böhrer, S. Brandt, G. Cowan, E. Feigl, C. Grupen, G. Lutters, J. Minguet-Rodriguez, F. Rivera, ${ }^{25}$ P. Saraiva, L. Smolik, F. Stephan,

Fachbereich Physik, Universität Siegen, 57068 Siegen, Fed. Rep. of Germany ${ }^{16}$

M. Apollonio, L. Bosisio, R. Della Marina, G. Giannini, B. Gobbo, G. Musolino, F. Ragusa ${ }^{20}$

Dipartimento di Fisica, Università di Trieste e INFN Sezione di Trieste, 34127 Trieste, Italy

J. Rothberg, S. Wasserbaech

Experimental Elementary Particle Physics, University of Washington, WA 98195 Seattle, U.S.A.

S.R. Armstrong, L. Bellantoni, ${ }^{30}$ P. Elmer, Z. Feng, D.P.S. Ferguson, Y.S. Gao, S. González, J. Grahl, T.C. Greening, J.L. Harton, ${ }^{28}$ O.J. Hayes, H. Hu, P.A. McNamara III, J.M. Nachtman, W. Orejudos, Y.B. Pan, Y. Saadi, M. Schmitt, I.J. Scott, V. Sharma, ${ }^{29}$ J.D. Turk, A.M. Walsh, Sau Lan Wu, X. Wu, J.M. Yamartino, M. Zheng, G. Zobernig

Department of Physics, University of Wisconsin, Madison, WI 53706, USA ${ }^{11}$

\footnotetext{
${ }^{1}$ Now at CERN, 1211 Geneva 23, Switzerland.

${ }^{2}$ Supported by Dirección General de Investigación Científica y Técnica, Spain.

${ }^{3}$ Now at Dipartimento di Fisica, Università di Lecce, 73100 Lecce, Italy.

${ }^{4}$ Also Istituto di Fisica Generale, Università di Torino, Torino, Italy.

${ }^{5}$ Also Istituto di Cosmo-Geofisica del C.N.R., Torino, Italy.

${ }^{6}$ Supported by the Commission of the European Communities, contract ERBCHBICT 941234.

${ }^{7}$ Supported by CICYT, Spain.

${ }^{8}$ Supported by the National Science Foundation of China.

${ }^{9}$ Supported by the Danish Natural Science Research Council.

${ }^{10}$ Supported by the UK Particle Physics and Astronomy Research Council.

${ }^{11}$ Supported by the US Department of Energy, grant DE-FG0295-ER40896.

${ }^{12}$ On leave from Universitat Autonoma de Barcelona, Barcelona, Spain.

${ }^{13}$ Supported by the US Department of Energy, contract DE-FG05-92ER40742.

${ }^{14}$ Supported by the US Department of Energy, contract DE-FC05-85ER250000.

${ }^{15}$ Permanent address: Universitat de Barcelona, 08208 Barcelona, Spain.

${ }^{16}$ Supported by the Bundesministerium für Forschung und Technologie, Fed. Rep. of Germany.

${ }^{17}$ Supported by the Direction des Sciences de la Matière, C.E.A.

${ }^{18}$ Supported by Fonds zur Förderung der wissenschaftlichen Forschung, Austria.

${ }^{19}$ Permanent address: Kangnung National University, Kangnung, Korea.

${ }^{20}$ Now at Dipartimento di Fisica, Università di Milano, Milano, Italy.

${ }^{21}$ Also at CERN, 1211 Geneva 23, Switzerland.

${ }^{22}$ Supported by the US Department of Energy, grant DE-FG03-92ER40689.

${ }^{23}$ Now at Wayne State University, Detroit, MI 48202, USA.

${ }^{24}$ Now at Lawrence Berkeley Laboratory, Berkeley, CA 94720, USA.

${ }^{25}$ Partially supported by Colciencias, Colombia.

${ }^{26}$ Also at Istituto di Matematica e Fisica, Università di Sassari, Sassari, Italy.

${ }^{27}$ Now at Schuster Laboratory, University of Manchester, Manchester M13 9PL, UK.

${ }^{28}$ Now at Colorado State University, Fort Collins, CO 80523, USA.

${ }^{29}$ Now at University of California at San Diego, La Jolla, CA 92093, USA.

${ }^{30}$ Now at Fermi National Accelerator Laboratory, Batavia, IL 60510, USA.
} 


\section{Introduction}

According to QCD, owing to their larger colour charge, gluon jets are expected to have softer particle energy spectra and to be wider than quark jets of the same energy. At leading order and asymptotic energies one expects the multiplicity ratio between pairs of back to back quark and gluon jets to be equal to the ratio of the Casimir factors $C_{A} / C_{F}=9 / 4$. At present energies this simple prediction is expected to be significantly altered by QCD coherence effects which strongly suppress the fragmentation of the gluon jet in the three-jet topology [1]. These predictions refer to the parton jets. Extrapolation to the final state hadrons relies on the Local Parton Hadron Duality assumption that the multiplicity of hadrons is proportional to the multiplicity of partons.

A number of experimental searches for differences between quark and gluon jets have been performed [2]. Until recently, $e^{+} e^{-}$studies did not utilise quark and gluon jets with equal energies, or jets produced in the same environment (e.g. they used gluon jets from three-jet events and quark jets from two-jet events). The interpretation of such comparisons is difficult and heavily Monte Carlo model dependent. LEP experiments [3] using three-jet events and exploiting high resolution vertex detectors have been able to identify gluon jets on an event by event basis. They were able to study quark and gluon jets with equal energies and from the same environment, so producing less model dependent analyses.

In this analysis the properties of $24 \mathrm{GeV}$ gluon and quark jets from one-fold symmetric three-jet events were studied. The gluon identification method is based on a $b$ anti-tagging procedure. The analysis relies on the assumption that the properties of gluon jets from $q \bar{q} g$ events are the same as in $b \bar{b} g$ events. The results presented here originate from two independent studies whose main difference was the type of $b$ tag employed; one used an impact parameter lifetime tag and the other a high transverse momentum lepton tag.

Two sets of comparisons were performed. The first one involved gluon tagged jets and quark jets whose flavour composition was determined by the electroweak couplings of the Z. The second comparison involved gluon tagged jets and $b$ jets. Gluon radiation from heavy quarks is restricted due to quark mass effects [4]. According to this, heavy flavour jets are expected to contain fewer particles than light quark and gluon jets. However the decay of the heavy hadron produces extra multiplicity [5] and hence the above prediction gets modified. The possible contrast between $b$ and gluon jets can be used to decide whether $b$ quark and gluon jets can be separated via general jet properties.

The properties studied are mean charged particle multiplicity, fragmentation function, rapidity distribution, and multiplicity and energy fraction within a given jet cone. The results obtained were compared with the Jetset $7.3[6]$ and Herwig 5.5 [7] model predictions, all subsequent mentions of these models refer to these explicit version numbers.

\section{The ALEPH detector}

The ALEPH detector is described in detail in [8]. A brief description of those parts of the apparatus most relevant to this analysis is presented here.

The tracking system consists of three subdetectors which are immersed in a magnetic field of $1.5 \mathrm{~T}$. Closest to the beam pipe is a high resolution vertex detector (VDET) consisting of two layers of double sided silicon microstrip detectors. The inner layer is at an average radius of $6.5 \mathrm{~cm}$ and covers $85 \%$ of the solid angle, whilst the outer 
layer is at $11.3 \mathrm{~cm}$ and covers $69 \%$ of the solid angle. The point resolution of the VDET is $12 \mu \mathrm{m}$ at normal incidence for both the $r \phi$ and $z$ dimensions. Surrounding the VDET is the inner tracking chamber, an eight layer axial drift chamber, and the time projection chamber (TPC) which provides up to 21 point measurements. Combined information from these three subdetectors yields a transverse momentum resolution of $\sigma\left(1 / p_{T}\right)=0.0006(\mathrm{GeV} / \mathrm{c})^{-1}$ measured with $45 \mathrm{GeV}$ muons; at low momentum multiple scattering dominates and adds a constant term of 0.005 to $\sigma\left(p_{T}\right) / p_{T}$. The TPC also provides up to 338 measurements of the specific ionization density $(\mathrm{dE} / \mathrm{dx}$ ) of a track.

The impact parameter resolution (for high momentum tracks) of $23 \mu \mathrm{m}$ and $28 \mu \mathrm{m}$ in the $r \phi$ and $z$ plane respectively [9], allowed this analysis to use the track impact parameter method to identify heavy flavour jets.

Outside the TPC is the electromagnetic calorimeter (ECAL) which consists of 45 layers of lead interleaved with proportional wire chambers and gives an energy resolution

$\sigma(\mathrm{E}) / \mathrm{E}=0.18 / \sqrt{\mathrm{E}(\mathrm{GeV})}$. Information from the ECAL, together with the $\mathrm{dE} / \mathrm{dx}$ measurements are used for electron identification. Muons are identified using the hadron calorimeter (HCAL) and the muon chambers. The HCAL is formed by the iron of the magnet return yoke interleaved with 23 layers of streamer tubes. It provides a twodimensional measurement of muon tracks and measurement of the hadronic energy. The HCAL is surrounded by two double layers of streamer tubes, the muon chambers, which provide three-dimensional information.

\section{Event Selection}

The standard ALEPH hadronic event selection [10] was applied to the 1990, 1991, and 1992 data ( $\approx 1$ million events) for the lepton-tag analysis and to the 1992 and 1993 data $\left(\approx 1.4\right.$ million events) for the lifetime-tag analysis. The $k_{\perp}$ (Durham) clustering algorithm [11], with $E$ recombination scheme and a jet resolution parameter of $y_{c u t}=0.01$ was applied to all charged and neutral particles (energy flow objects [9]) to select three-jet events. All jets were required to have a polar angle greater than $30^{\circ}$ with respect to the beam axis. In the case of the impact parameter analysis, this was increased to $40^{\circ}$ for the two lower energy jets so that they were well within the solid angle covered by the vertex detector.

The jets were projected onto the event plane which was defined according to the quadratic momentum tensor. One-fold symmetric configurations were selected by requiring that the angles in the event plane between the highest energy jet and each of the two lower energy jets were in the range $150^{\circ} \pm 7.5^{\circ}$ in the case of the impact parameter $\operatorname{tag}$ and $150^{\circ} \pm 10^{\circ}$ in the case of the lepton-tag. This kinematic configuration implied that the mean energy of each of the two lower energy jets was $24.7 \mathrm{GeV}$ for quark jets and $24.0 \mathrm{GeV}$ for gluon jets. From the 1992 and 1993 data 10447 events satisfied the one-fold symmetric criteria.

The Monte Carlo events analysed were generated using the Jetset parton shower model with $b$ and $c$ quark fragmentation given by the Peterson et al. parameterisation [12]. They were passed through the full ALEPH detector simulation and, having satisfied the above selection criteria, were analysed in the same way as the data. 


\section{Analysis Method}

The symmetric event configuration has been previously used in various analyses of quark and gluon jets $[3,13]$. It is advantageous in that it ensures that the quark and gluon jets have almost the same energy. The one-fold symmetric configuration employed by the present analysis guarantees a large energy difference between the most energetic jet (J1) and the two other (J2, J3). Hence $\mathrm{J} 1$ has a high probability of originating from a quark (or anti-quark). The Monte Carlo estimate is that in only $3 \%$ of the events $\mathrm{J} 1$ is a gluon jet.

The mixture of $\mathrm{J} 2$ and $\mathrm{J} 3$ jets from each event constituted the mixed sample, M, containing almost half quark and half gluon jets of equal energies. The quarks are a mixture of flavours determined by the electro-weak couplings of the $\mathrm{Z}$ referred to as the natural flavour mix, NFM. If for each event, one of the two lower energy jets has a high probability to be a $b$ jet, the remaining jet is identified as a gluon jet and enters into the gluon tagged sample, T.

The analyses are based on the comparison of the $\mathrm{M}$ and $\mathrm{T}$ samples which allows the study of jets which have had no tagging criteria applied directly to them, hence the bias introduced by the tagging method is kept to a minimum.

\subsection{Purity Evaluation}

Pure quark and gluon jet properties can be extracted from the $\mathrm{M}$ and $\mathrm{T}$ samples via a simple unfolding procedure if the gluon purity of the $\mathrm{T}$ sample is known. This purity, defined as the ratio of the number of correctly tagged gluon jets over the number of jets tagged as gluon jets, was estimated from Monte Carlo events using a procedure to relate each reconstructed jet to its parent parton.

Assigning the particles produced during hadronisation to a specific causal parton is unambiguous only within an independent fragmentation scheme. However, as the final state hadrons form three well separated jets one expects the final state partons, i.e. all partons present at the end of the parton shower, to also cluster into three jets. An evaluation of the jet purity based on this approach requires three stages.

First, the final state partons were forced to cluster into three jets. Then, the three jets were classified as quark or gluon jets. The quark/antiquark jet was defined as the parton jet containing the original quark/anti-quark from the $\mathrm{Z}$ decay. If the original quark and anti-quark were found in two separate parton jets, as was normally the case, the third jet was interpreted as originating from a hard gluon. The case in which both quark and anti-quark were found in the same parton jet was found to occur in less than $1 \%$ of the symmetric events and these events were therefore ignored in the purity study. Finally, the parton-level jets were matched to the detector-level jets according to the following scheme. The pair of parton-level and detector-level jets with the smallest angular separation was identified first. Of the remaining two combinations, the one that minimized the sum of the angular separations was chosen.

In the purity definition the number of correctly tagged gluon jets corresponds to the number of tagged gluon jets that were matched to a parton-level gluon jet according to the above procedure. For the chosen value of $y_{c u t}(0.01)$, the mean angular separation between the parton-level gluon jet and the matched detector-level gluon jet was found to be $5^{\circ}$. This value of $y_{\text {cut }}$ was chosen as the best compromise to minimise ambiguous jet association whilst maintaining a $\approx 20 \%$ three-jet rate. 


\subsection{The Tagging Procedure}

By identifying one of the two lower energy jets as a $b$ jet, the other is anti-tagged as the gluon jet of the event. Jets originating from a $b$-quark can often be identified via the characteristics of the decay of a $B$ hadron: the presence of a secondary vertex or the presence of a high transverse momentum lepton.

\subsubsection{The Lifetime Tag}

As the aim is the identification of the $b$-jet and not the reconstruction of the final $B$ state, explicit vertex reconstruction is not necessary. An impact parameter identification method can take advantage of the presence of a second and third vertex formed by the decay of a long-lived $D$-hadron originating from the cascade decay $B \rightarrow D$. The lifetime tag algorithm used [14] combines information from all tracks to form the jet probabilities, $P_{i}$, for the hypothesis that all tracks of jet Ji come from the primary vertex. A jet (J3) was tagged as a gluon jet and included in the $\mathrm{T}$ sample if the other jet (J2) had a high probability of being a $b$-jet: $P_{2}<P_{\text {cut }}=0.002$.

The algorithm is designed to avoid correlations between the tag variables of the jets. This is important for the analysis as such correlations could introduce biases in the properties of the $b$ anti-tagged gluon sample. Nevertheless high multiplicity $b$ decays are tagged more efficiently. Although the effect of this correlation is small, a careful study of varying $P_{c u t}$ was performed (with MC events) to understand and minimize any consequences for the jet properties. The track multiplicity of the correctly tagged gluons was found to be smaller than the equivalent value measured on an untagged sample. This bias depends on the value of $P_{c u t}$ used. The flavour composition of the misidentified quark jets of the T sample also depends on the $P_{c u t}$ value. This composition should be the same as the corresponding flavour composition of the $\mathrm{M}$ sample so that the quark and gluon jets from the $\mathrm{M}$ and $\mathrm{T}$ samples can be compared. Consistent results for various values of $P_{\text {cut }}$ were obtained when including bias correction factors, see 4.3, eq.(4). For the final analysis the tagging procedure was optimized for the highest gluon purity and smallest bias in the jet properties. The $\mathrm{T}$ sample used consisted of 1002 jets with an estimated gluon purity $P_{g}^{T}=0.90$.

\subsubsection{The Lepton Tag}

The presence of a high transverse momentum $\left(P_{\perp}\right)$ lepton has been widely used in the tagging of heavy flavour events and a standard lepton selection definition [15] exists within the ALEPH collaboration. Electrons and positrons were selected using the $\mathrm{dE} / \mathrm{dx}$ capabilities of the TPC and estimators based on the shape of the energy depositions in the ECAL. Muons were selected according to digital hit information recorded by the HCAL and the muon chambers. The minimum momentum of any selected lepton was $3 \mathrm{GeV} / \mathrm{c}$. In turn, the momentum of each selected lepton was removed from its jet and its $P_{\perp}$ was calculated with respect to the resulting jet axis. This is known as the exclusive transverse momentum $\left(P_{\perp}^{e x c l}\right)$ of the lepton. In order to tag a jet as a heavy quark jet it was required to contain a lepton whose $P_{\perp}^{e x c l}$ was greater than $0.5 \mathrm{GeV} / \mathrm{c}$.

By finding a high $P_{\perp}$ lepton in one of the two lower energy jets, the gluon-tagged sample, T, was obtained. This sample contained 745 jets with an estimated gluon purity of $82 \%$. In contrast to the impact parameter tagging method, the presence of a lepton in one (say J2) jet is mostly uncorrelated with the properties of the other (gluon) jet. Hence 
gluon jet properties of the lepton tagged $\mathrm{T}$ sample do not suffer from systematic tagging biases. This was confirmed with Monte Carlo studies.

The lepton study also analysed a mixed sample of gluon and $b$ jets of $24 \mathrm{GeV}$ energy, referred to as the $\mathrm{B}$ sample, selected by finding a high $P_{\perp}$ lepton in the highest energy jet. The two lower energy jets were then taken as equally likely to be the other $b$ jet or the gluon jet. The B sample contained 806 events with $b$ purity of $66.5 \%$.

\subsection{Unfolding of the Jet Properties}

Figure 1 shows the three sample types used. The mixed sample, M, consisted of the two lower energy jets from all symmetric events. The tagged sample, T, consisted of the gluon-tagged jets from the events in which one of the two lower energy jets was identified as a $b$ jet. The $\mathrm{B}$ sample consisted of the two lower energy jets from events for which the highest energy jet was identified as a $b$ jet. In the case of the impact parameter analysis only the $\mathrm{M}$ and $\mathrm{T}$ samples were considered. The $\mathrm{B}$ sample was identical to the $\mathrm{M}$ sample in every respect other than the quark flavour mix. Hence this section only discusses the unfolding of the $\mathrm{M}$ and $\mathrm{T}$ samples. Results from the comparison of the $\mathrm{T}$ and $\mathrm{B}$ samples were extracted in exactly the same way and are discussed in later sections.

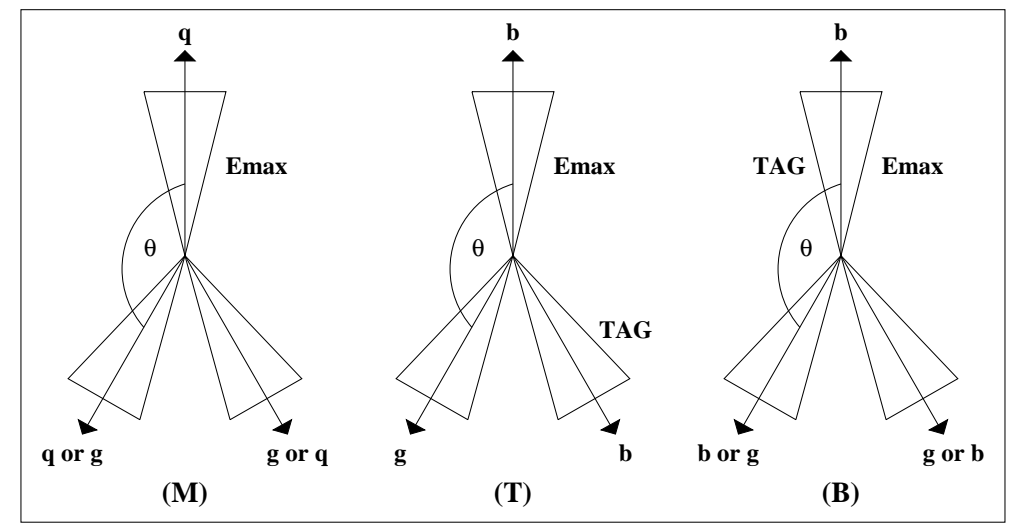

Figure 1: The three tagging configurations: the 50\% quark-gluon (M)ixed sample, the gluon (T)agged sample, and the (B)-quark enriched mixed sample.

Consider a gluon-tagged $\mathrm{T}$ sample whose purity has been estimated to be $P_{g}^{T}$. Let $A$ be the observable under study, and $A_{g(q)}$ the corresponding value for pure gluon (quark) jets. The value $A^{T}$ measured from the $\mathrm{T}$ sample may then be expressed as

$$
A^{T}=P_{g}^{T} A_{g}+\left(1-P_{g}^{T}\right) A_{q} .
$$

The corresponding equation for an $M$ sample of quark and gluon jets with a gluon component $P_{g}^{M}$, different from $P_{g}^{T}$ is

$$
A^{M}=P_{g}^{M} A_{g}+\left(1-P_{g}^{M}\right) A_{q} .
$$

Knowing $P_{g}^{T, M}$, eqs. $(2,3)$ can be solved for $A_{g}$ and $A_{q}$, allowing a direct comparison of the properties of quark and gluon jets.

Ideally the two samples should consist of events where the jets are produced in the same kinematical configurations and the tagging procedure should not introduce a bias. While (almost) identical kinematics are ensured by using symmetric events, the existence of a tagging bias cannot be excluded. Using Monte Carlo events, the impact parameter 
analysis estimated this bias by comparing the value of the observable $A_{g(q)}$ from the sample of all gluon (quark) jets in the symmetric configuration (i.e., from the $\mathrm{M}$ sample) to the value $A_{g(q)}^{\text {Bias }}$ measured from correctly identified gluon (quark) jets from the $\mathrm{T}$ sample. Defining the tagging bias, $\Delta A$, as:

$$
\Delta A_{g(q)}=1-\frac{A_{g(q)}^{\text {Bias }}}{A_{g(q)}},
$$

eq. (1) becomes

$$
A^{T}=P_{g}^{T} A_{g}\left(1-\Delta A_{g}\right)+\left(1-P_{g}^{T}\right) A_{q}\left(1-\Delta A_{q}\right) .
$$

Equation(2) remains the same as no tag was applied to the M sample. These correction factors were evaluated using Jetset events and were in general small (less than $2 \%$ ). Similar factors extracted from Herwig events were found to be compatible. In the case of the gluons from the lepton-tag analysis, $\Delta A$ was measured to be zero within the Monte Carlo statistics, as already mentioned in the previous section.

\subsection{Detector Effects}

The unfolded results were finally corrected for detector effects. These were estimated by comparing the properties of quark and gluon jets generated by the Jetset model before and after detector simulation. These corrections carried statistical and systematic (model dependent) errors. The latter were estimated by comparing the detector correction factors extracted from the Jetset and Herwig models.

\section{$5 \quad$ Measured Quark and Gluon Jet Properties}

The lifetime-tag analysis measured properties of $24 \mathrm{GeV}$ energy gluons and quarks with a natural flavour mix (NFM), embedded in symmetric three jet events. The lepton-tag analysis measured in addition the properties of $b$ quarks of the same energy and from the same environment. The two analyses yielded very similar results for the gluon and NFM quark properties. Hence in the following section results of the gluon and NFM quark jet properties from the two analyses are not considered separately. The discussion of quarkgluon jet comparisons refers to results of the lifetime-tag analysis while the comparison of $b$ and gluon jets is based on results of the lepton-tag analysis.

The lifetime-tag analysis also tested the unfolding method by modifying the $P_{c u t}$ to obtain and compare an $80 \%$ pure gluon T-sample with the mixed M-sample. The two sets of corrected distributions (from the $90 \%$ and $80 \%$ pure gluon T-sample) were found to be in very good agreement.

\subsection{Systematic Uncertainties}

The results discussed in the next subsections are presented with statistical and systematic errors. The systematic errors include the following contributions:

1. The statistical accuracy of the gluon purity value extracted from Jetset. This was practically negligible. 
2. The systematic error in the gluon purity which arises from the Monte Carlo model and the purity evaluation algorithm dependencies. This error was estimated using Herwig as an alternative model and by evaluating theoretically (to first order) the relevant quark and gluon purity in the $\mathrm{M}$ sample. The contribution of this systematic error to the various measurements was below $1 \%$.

3. The error associated with the tagging procedure bias. This was more significant, being of the order of a few percent ( $1-4 \%$ for the central region of the distributions).

4. The error associated with the detector effects corrections. This was already discussed in section 4.4 and was also approximately a few percent $(2-5 \%$ for the central region of the distributions).

The last two systematic errors were evaluated by extracting the relevant correction factors from Herwig events and comparing them with those extracted from Jetset events. In each case the assigned systematic error was equal to either the difference between the two sets of correction factors or the statistical error on the Herwig correction, whichever was the largest.

\subsection{Mean Charged Particle Multiplicity}

The lifetime-tag analysis gave the following results. The raw charged particle multiplicities measured for the jets in the $\mathrm{T}$ and $\mathrm{M}$ samples are $\left\langle n_{c h}^{T}>=8.26 \pm 0.10\right.$ and $<n_{c h}^{M}>=7.70 \pm 0.02$, respectively. The unfolded values, corrected for bias and detector effects are $\left\langle n_{c h}\right.$ (gluon) $\rangle=9.94 \pm 0.14$ (stat) \pm 0.27 (syst) and $\left\langle n_{\text {ch }}\right.$ (quark) $\rangle=$ $8.37 \pm 0.15$ (stat) \pm 0.22 (syst). The corresponding ratio is given by:

$$
R_{c h} \equiv \frac{<n_{c h}(\text { gluon })>}{<n_{c h}(\text { quark })>}=1.19 \pm 0.04(\text { stat }) \pm 0.02(\text { syst }) .
$$

The lepton-tag analysis yielded a compatible measurement for $R_{c h}$. This result deviates significantly from unity in agreement with recent LEP results [3], confirming the higher gluon multiplicity. It is significantly lower than the naive asymptotic prediction of $C_{A} / C_{F}=9 / 4$.

The Jetset Monte Carlo predicts $<n_{c h}($ gluon $\left.)\right\rangle=10.16$ and $\left\langle n_{c h}(\right.$ quark $\left.)\right\rangle=7.92$ leading to a ratio of $R_{c h}=1.28$ (with negligible statistical errors). The same analysis was done with the Herwig Monte Carlo model. The resulting values are $<n_{c h}($ gluon $)>=9.48$, $<n_{c h}(q u a r k)>=7.63$ and $R_{c h}=1.24$. Finally the multiplicity ratio was determined at the parton-level of the Jetset Monte Carlo. The result, $R_{\text {part }}=1.29$, is again very similar with the hadron-level result, suggesting that the observed difference between quark and gluon jets does have a perturbative origin.

The lepton-tag analysis measured the ratio of gluon to $b$ jet multiplicity. It was found consistent with unity:

$$
\frac{\left\langle n_{c h}(\text { gluon })\right\rangle}{\left\langle n_{c h}(\text { bquark })\right\rangle}=1.00 \pm 0.05(\text { stat }) \pm 0.02(\text { syst })
$$

indicating that for the energy scales involved, the additional particle multiplicity arising from the $B$ hadron decay masks completely the difference between $b$ quark and gluon jet multiplicity. This effect is present in Jetset, estimating the corresponding ratio $R_{c h}=1.077$. This measurement is also in agreement with the recent result from OPAL [16]. 


\subsection{Fragmentation Function}

Figure 2 shows the unfolded and corrected fragmentation function, $(1 / N) d N / d \mathrm{X}_{\mathrm{E}}$, with $\mathrm{X}_{\mathrm{E}}=\mathrm{E}_{\text {particle }} / \mathrm{E}_{\mathrm{jet}}$, for charged particles measured by the lifetime-tag analysis together with the estimates of the Jetset and Herwig Monte Carlo models. Gluon jets have more particles carrying small fractions of the total energy, i.e. they are softer. The differential distributions presented in figure 2 are also given in table 1.

\subsection{Jet Shape Variables}

The rapidity distribution is another way of looking at the multiplicity and shape of a jet. Gluon jets, having greater multiplicity, are expected to have a higher plateau; the ratio of the heights of the corresponding distributions of gluon and quark jets is expected to asymptotically tend to $C_{A} / C_{F}$. Moreover, coherence effects, in conjunction with the selection of the events as three-jet events according to a specific jet algorithm, are expected to suppress the length of the gluon plateau [1], yielding a narrower rapidity distribution. Figure 3 shows the measurements of the lifetime-tag analysis for the rapidity distributions of charged particles (assuming the pion mass) of the two types of jet, normalised to the total number of jets. They confirm the theoretical predictions. The rapidity region $Y<1$ corresponds to angles greater than $40^{\circ}$ from the jet axis. As the angle between the two lower energy jets is $\approx 60^{\circ}$ this rapidity region is difficult to interpret. The heights of the quark and gluon distributions were estimated by fitting the relevant distributions with double Gaussians, which were found to satisfactorily describe the data. The ratio of the heights of the gluon and quark rapidity plateaus is measured to be $1.45 \pm 0.15$, i.e. higher than the corresponding multiplicity ratio. These measurements are in qualitative agreement with Monte Carlo predictions from the Jetset and Herwig models, as can be seen from figure 3 .

Figure 4 compares the gluon rapidity distribution with the corresponding property for $b$ jets as measured by the lepton-tag analysis. The two distributions are now very similar indicating that the $B$ hadron decay, for the current energies, makes $b$ jets similar to gluon jets. Jetset reproduces well the $b$ jet distribution.

Another way of illustrating the broadness of a jet is to study the number of particles and the fraction of energy found within a cone around the jet axis. This is equivalent to integrating the rapidity distribution. Figure 5 shows the integrated charged particle multiplicity and figure 6 shows the integrated energy fraction as a function of the cone size around the jet axis for NFM quark and gluon jets measured by the lifetime-tag analysis together with the corresponding estimates of Jetset and Herwig. Gluon jets are clearly broader. Although gluon jets have higher total multiplicity, quark jets contain more particles within a cone of half angle up to $\approx 15^{\circ}$. Quark jets have on average about $32 \%$ of their energy enclosed within a half cone of $5^{\circ}$, compared to only $16 \%$ for gluon jets. In general the models reproduce the data well. The plots also contain data obtained by the lepton-tag analysis which measured the multiplicity and energy fraction of $b$ jets as a function of the cone half angle. From figure 5 one sees that $b$ jets have a higher multiplicity than light quarks, as expected.

\section{Summary and conclusions}

Three jet events of symmetrical topology were chosen as a clean and simple environment to study and compare gluon and quark jets of $24 \mathrm{GeV}$. Two $b$ jet tagging techniques were 
used to identify quark jets and hence anti-tag gluon jets, introducing minimal biases to the jet properties. The two $b$-tag identification methods gave very similar results. Significant differences between quark and gluon jets were observed. Gluon jets were found to have a higher multiplicity than quark jets. In addition gluon jets were shown to have a softer fragmentation function and to be less collimated around the jet axis than quark jets, as qualitatively expected by QCD. The difference in jet shape is clearly displayed both by the particle and energy distribution within successive cones around the axis and by the rapidity distributions which exhibit the behaviour recently predicted and associated with colour coherence. Both the Jetset and Herwig model predictions are in agreement with these results. The analysis of a $b$ jet sample showed that the above differences are markedly reduced if gluon jet properties are compared to the corresponding $b$ jet properties, implying that, for the current energy scale, the non-leading fragmentation of the heavy flavour quark contributes significantly to the development of the jet profile.

\section{Acknowledgements}

We wish to thank our colleagues in the CERN accelerator division for the excellent performance of the LEP machine. Thanks are also due to the engineers and technicians at all the collaborating institutions for their contribution to the success of ALEPH. Those of us from non-member states are grateful to CERN for its hospitality. 


\section{References}

[1] S.Catani etal., Nucl. Phys. B383 (1992)419

[2] W. Bartel et al., JADE Collaboration, Z. Phys. C21 (1983)37;

Y.K.Kim etal., AMY Collaboration, KEK 90-79;

P. Bagnaia et al., UA2 Collaboration, Phys. Lett. B144 (1984)291;

M.Derrick et al., HRS Collaboration, Phys. Lett. B165 (1985)449

[3] M.Z. Akrawy et al., OPAL Collaboration, Phys. Lett. B261 (1991)334;

R. Akers, OPAL Collaboration, CERN-PPE/95-075 sub. to Z. Phys. C;

D.Buskulic et al., ALEPH Collaboration, Phys. Lett. B346 (1995)389.

[4] V.A.Khoze, 26th International Conference on High-Energy Physics, Dallas 1992 Proceedings Ed. by J.R.Sanford

[5] P.C. Rowson etal., Mark II Collaboration, Phys. Rev. Lett.54 (1985)2580.

[6] T. Sjöstrand, Comp. Phys. Comm. 39 (1986)347;

T. Sjöstrand and M. Bengtsson, Comp. Phys. Comm. 43 (1987)367.

[7] G. Marchesini etal., Comp. Phys. Comm. 67 (1992)465.

[8] D.Decamp etal., ALEPH Collaboration, Nucl. Instr. Meth. A294 (1990)121.

[9] D.Buskulic et al., ALEPH Collaboration, Nucl. Instr. Meth. A360(1995) 481.

[10] D.Decamp etal., ALEPH Collaboration, Phys. Lett. B231 (1989)519.

[11] Y.L.Dokshitzer, contribution to "Workshop on Jets at LEP and Hera", Durham(1990);

S. Catani et al, Phys. Lett. B269(1991) 432.

[12] C.Peterson etal., Phys. Rev. D27 (1983)105.

[13] M.Derrick et al., HRS Collaboration, Phys. Lett. B165 (1985)449;

P.Abreu et al., DELPHI Collaboration, Z. Phys. C56(1992)63

[14] D.Buskulic et al., ALEPH Collaboration, Phys. Lett. B313 (1993)535.

[15] D.Buskulic et al., ALEPH Collaboration, Nucl. Instr. Meth. A346 (1994)461

D.Buskulic et al., ALEPH Collaboration, Z. Phys. C62(1994)179.

[16] G.Alexander et al., OPAL Collaboration, CERN-PPE/95-126 sub. to Z. Phys. C. 


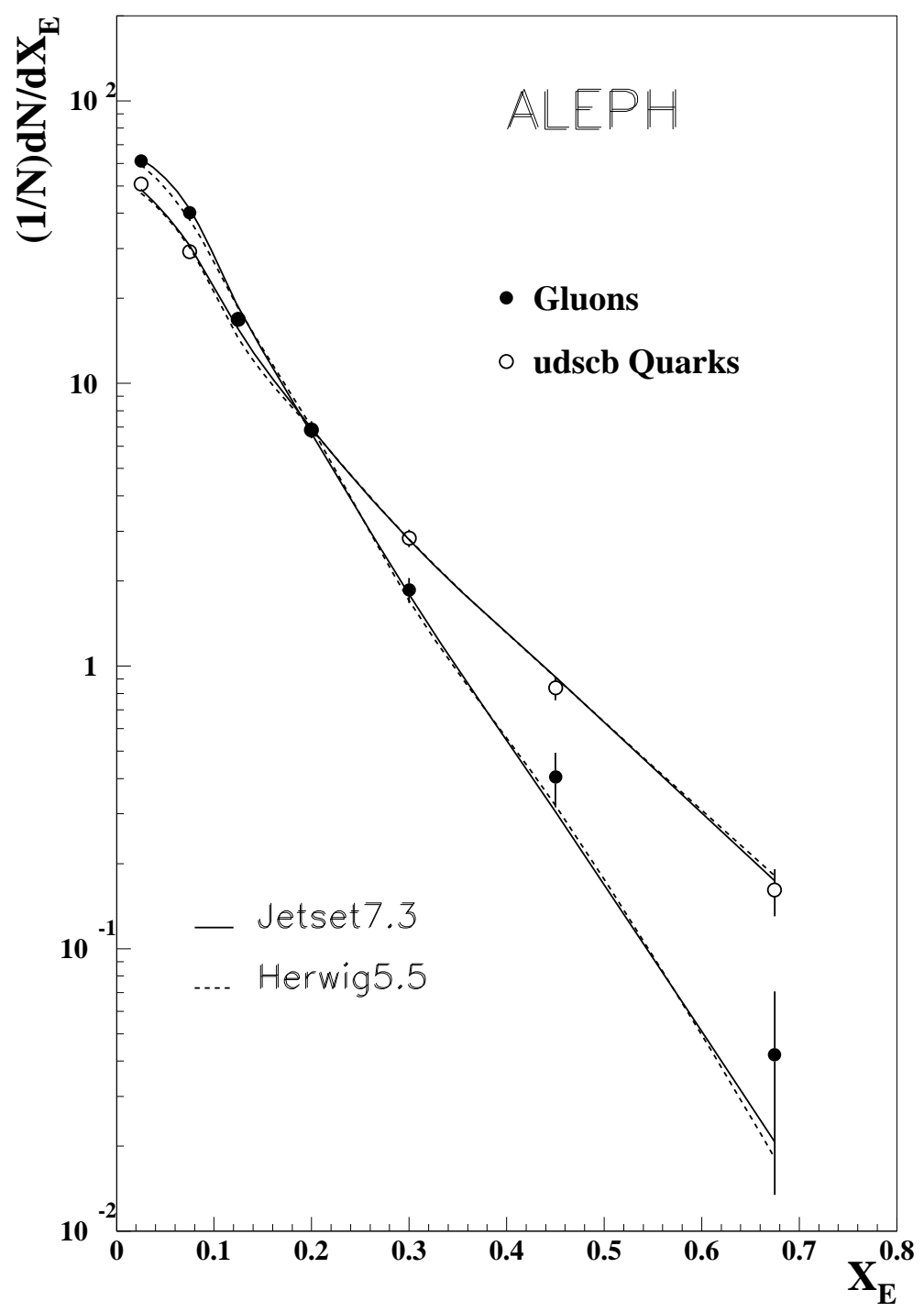

Figure 2: Fragmentation function for natural flavour mix quark and gluon jets.

Table 1: Fragmentation function for natural flavour mix quark and gluon jets.

\begin{tabular}{|c|c|c|}
\hline$X_{E}$ & \multicolumn{1}{|c|}{ Quarks } & \multicolumn{1}{c|}{ Gluons } \\
\hline $0.00-0.05$ & $50.86 \pm 1.67$ & $61.19 \pm 2.24$ \\
\hline $0.05-0.10$ & $29.35 \pm 1.49$ & $40.22 \pm 1.46$ \\
\hline $0.10-0.15$ & $16.91 \pm 0.96$ & $16.87 \pm 0.91$ \\
\hline $0.15-0.25$ & $6.84 \pm 0.40$ & $6.87 \pm 0.47$ \\
\hline $0.25-0.35$ & $2.83 \pm 0.20$ & $1.86 \pm 0.19$ \\
\hline $0.35-0.55$ & $0.83 \pm 0.08$ & $0.40 \pm 0.09$ \\
\hline $0.55-0.80$ & $0.16 \pm 0.03$ & $0.04 \pm 0.03$ \\
\hline
\end{tabular}




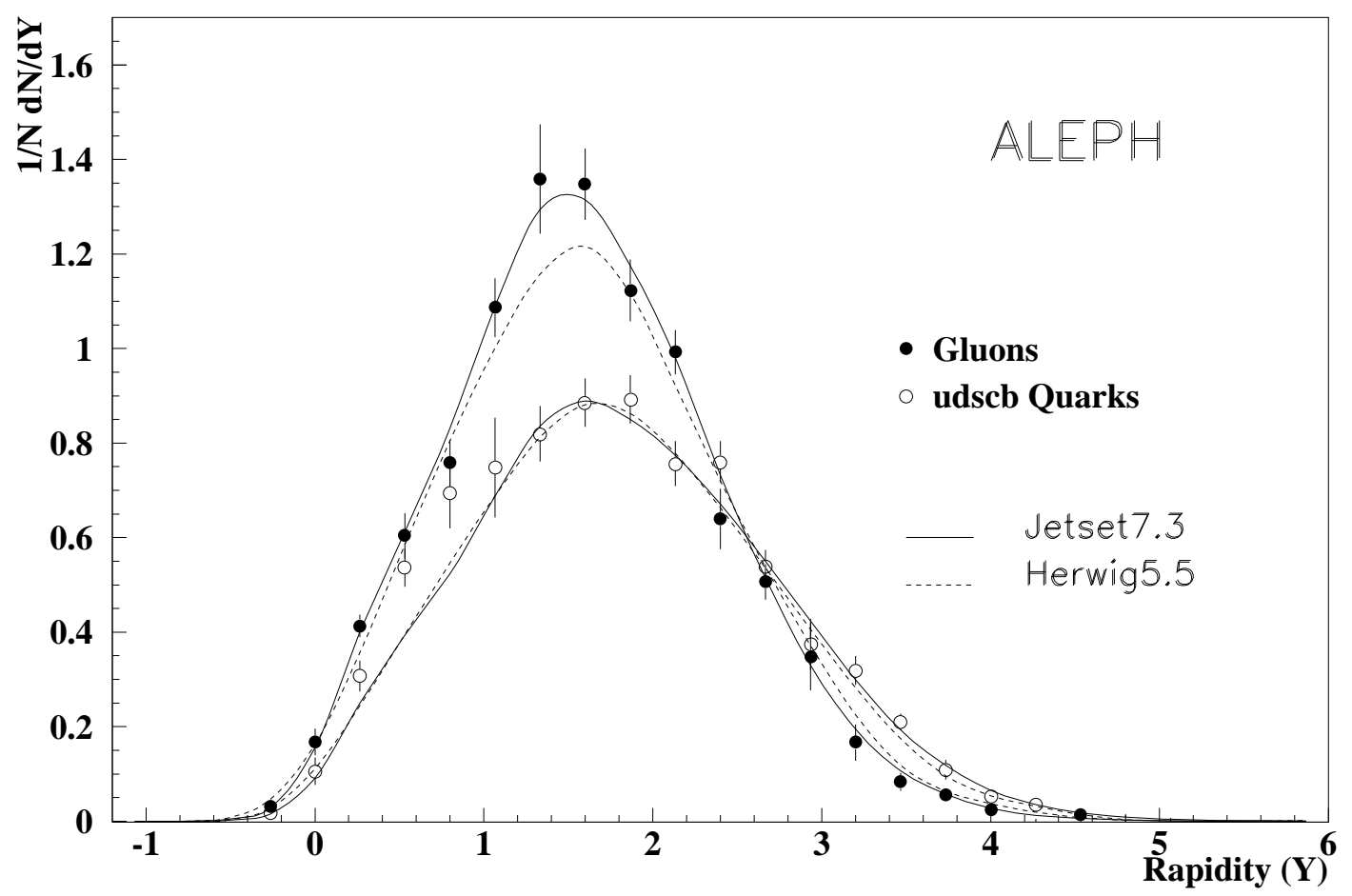

Figure 3: Rapidity distributions for natural flavour mix quark and gluon jets.

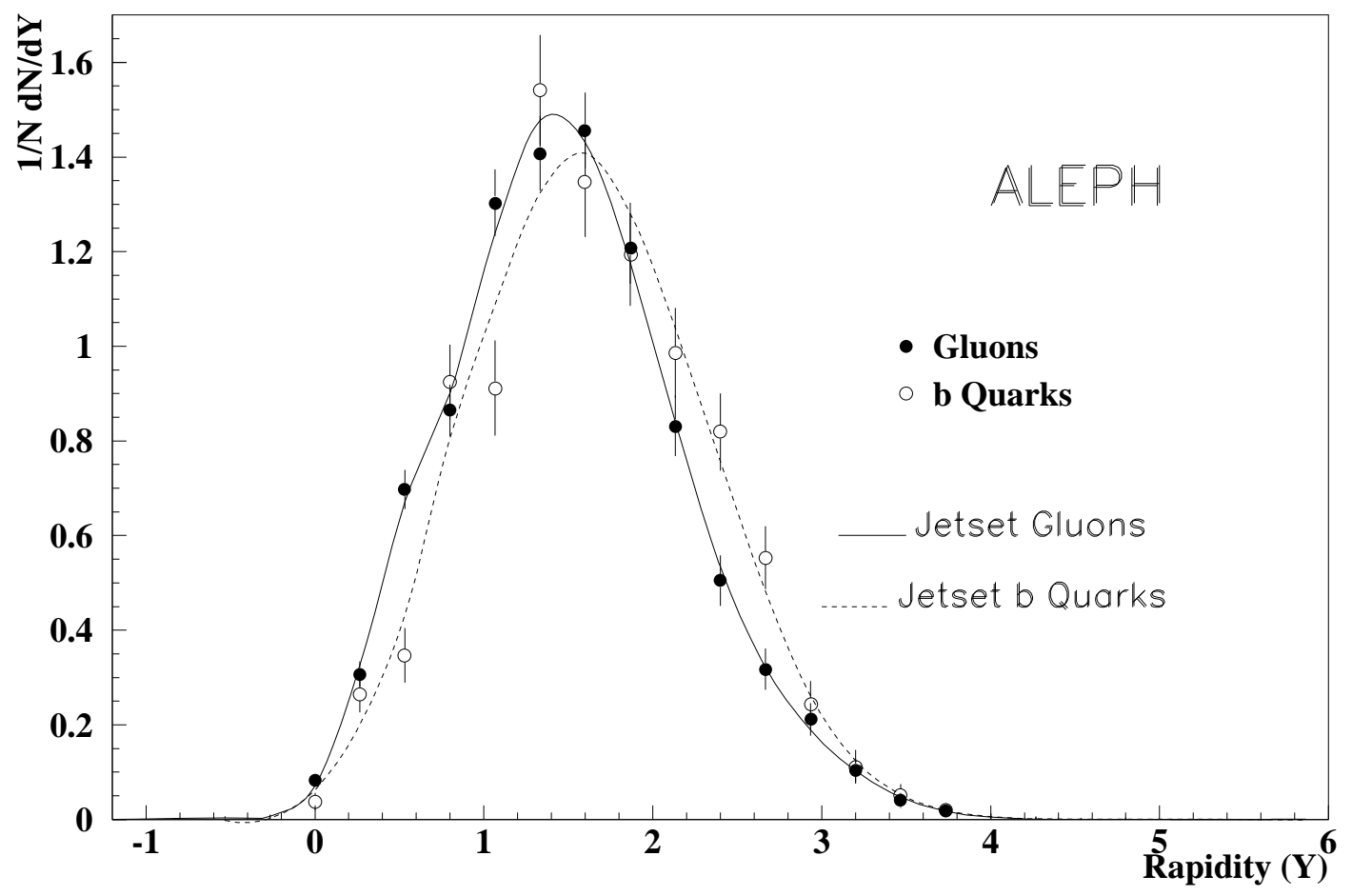

Figure 4: Rapidity distributions for $b$ quark and gluon jets. 


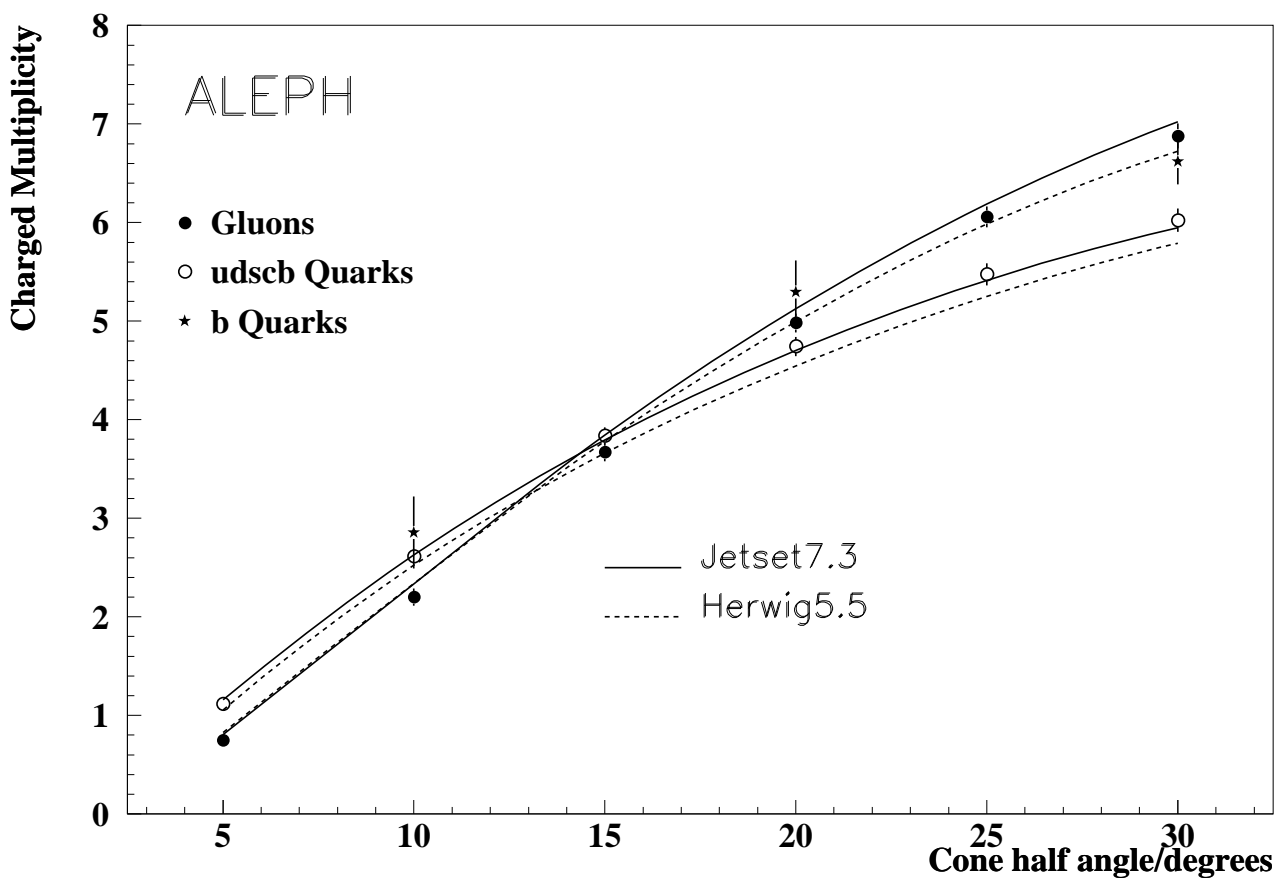

Figure 5: Integrated charged multiplicity within successive cones for quark and gluon jets.

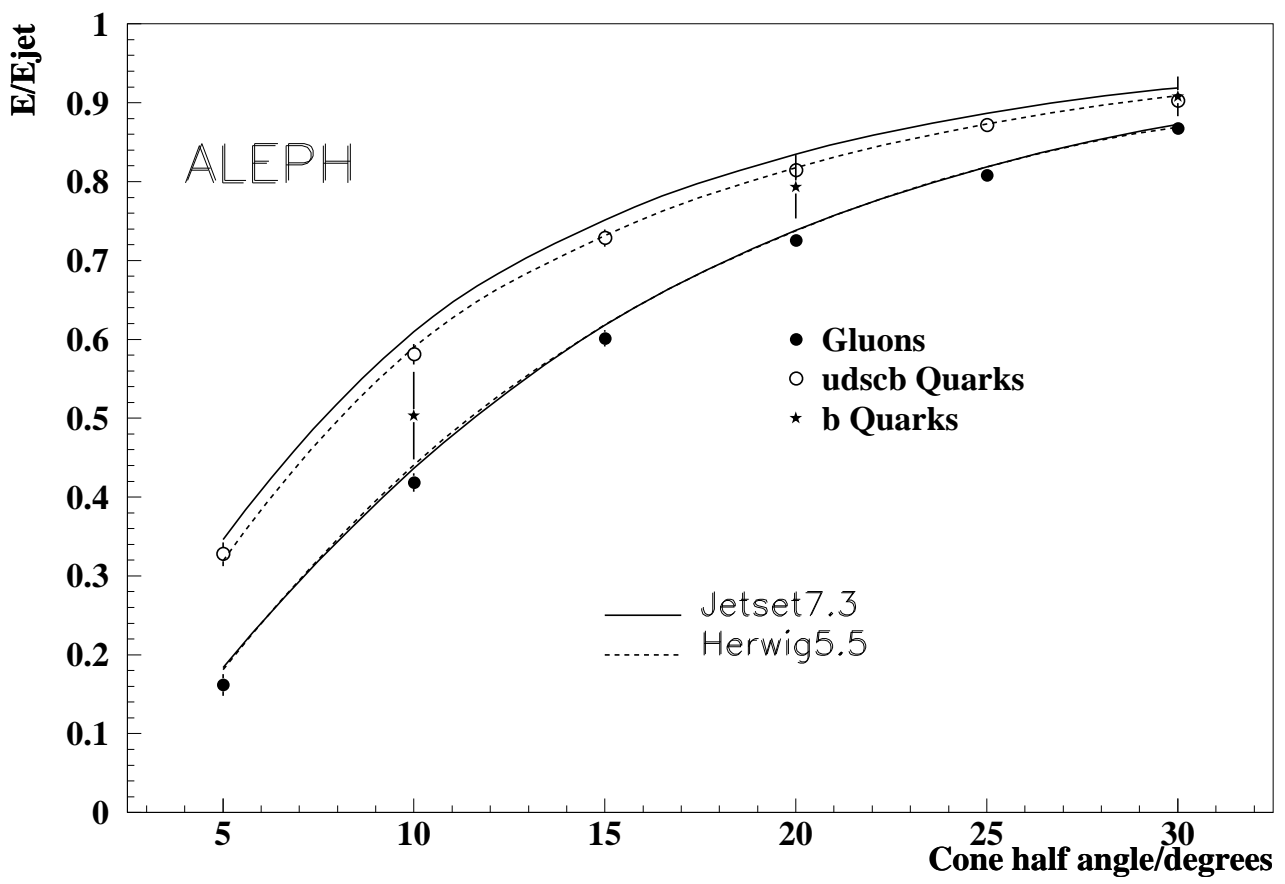

Figure 6: Integrated energy fraction within successive cones for quark and gluon jets. 